\title{
Predictive Vehicle Route Optimization in Intelligent Transportation Systems
}

\author{
Mohamad Abdul-Hak ${ }^{1,}$, , Nizar Al-Holou ${ }^{2}$, Youssef Bazzi ${ }^{3}$, Malok Alamir Tamer ${ }^{2}$ \\ ${ }^{1}$ Mercedes Benz Research and Development North America Inc., Redford, Michigan, USA \\ ${ }^{2}$ Department of Electrical \& Computer Engineering, University of Detroit Mercy Detroit, Michigan, USA \\ ${ }^{3}$ Faculty of Engineering, Lebanese University, Beirut, Lebanon
}

\section{Email address:}

mohamad.abdul-hak@daimler.com (M. Abdul-Hak), alholoun@udmercy.edu (N. Al-Holou), dryoussefabazzi@gmail.com (Y. Bazzi), may.blue.m@gmail.com (M. A. Tamer)

${ }^{*}$ Corresponding author

\section{To cite this article:}

Mohamad Abdul-Hak, Nizar Al-Holou, Youssef Bazzi, Malok Alamir Tamer. Predictive Vehicle Route Optimization in Intelligent Transportation Systems. International Journal on Data Science and Technology. Vol. 5, No. 1, 2019, pp. 14-28.

doi: $10.11648 /$ j.ijdst.20190501.13

Received: March 7, 2019; Accepted: April 26, 2019; Published: May 20, 2019

\begin{abstract}
Through the adoption of dedicated short-range communication (DSRC) wireless communication technology, intelligent transportation systems (ITS) will spur a new revolution in the U.S. transportation system. This paper is structured around providing drivers with the least-congested transportation route choices enabled by the ITS-envisioned vehicle-to-vehicle (V2V), vehicle-to-infrastructure (V2I), and infrastructure-to-vehicle (I2V) communication platforms. Recent research in vehicle navigation systems has proposed energy consumption and emission optimized routing methodologies using historical traffic data modeling. More than $50 \%$ of congestion in U.S. cities is nonrecurring congestion. Nonrecurring congestion reduces the availability of the traffic network, thus rendering historical traffic data-based systems insufficient in more than $50 \%$ of the cases. Real-time traffic data modeling provides an enhanced performance in traffic congestion assessment; however, greater performance is expected with a predictive traffic congestion model with increased certainty. This paper compares the conventional shortest path and fastest path vehicle routing methodologies and establish the improvement for environmentally friendly routing in a dynamic and predictive cost dependent traffic network based on Petri Net Modeling. The proposed routing algorithm is validated using a computer-based tool of choice.
\end{abstract}

Keywords: Intelligent Transportation Systems (ITS), Predictive Traffic Information, Environmentally Friendly Navigation, Emission, Dedicated Short-Range Communication (DSRC)

\section{Introduction}

Earth's Atmosphere naturally consists of greenhouse gases (GHGs), which enable the transmission of visible light and the absorption of infrared radiation, thus passing solar radiation from the Sun and trapping the heat energy within Earth's atmosphere. Without the balanced GHG effect, the planet would not be warm enough to sustain life in the form known to us today. Carbon dioxide $\left(\mathrm{CO}_{2}\right)$ is the largest contributor to the overall GHG concentration, with a share that has grown by $29 \%$ from 1959 to 2018 [1]. In 1896, Arrhenius was the first to mathematically correlate increased $\mathrm{CO}_{2}$ concentration in the atmosphere to Earth's increased surface temperature [2]. In
February 2007, 111 years later, thousands of scientific researchers on the Intergovernmental Panel on Climate Change (IPCC) collectively concluded that industrialization is causing global warming through the acceleration of $\mathrm{CO}_{2}$ emissions. Increased temperature on Earth's surface is causing extreme weather changes, such as droughts, floods, heavy rain, and excessive heat, resulting in fires. We can reduce future climate change threats by investing in technologies that reduce greenhouse gas emissions.

Domestic transportation $\mathrm{CO}_{2}$ emissions increased by 23 percent (332.8 MMT $\mathrm{CO}_{2}$ ) between 1990 and 2017, an annualized increase of 0.8 percent. Among domestic transportation sources in 2017, light-duty vehicles (including 
passenger cars and light-duty trucks) represented 58 percent of $\mathrm{CO}_{2}$ emissions from fossil fuel combustion, medium- and heavy-duty trucks and buses 25 percent. It has become clear that the vehicle industry, contributing $83 \%$ of all $\mathrm{CO}_{2}$ emissions from the transportation industry, is a key consideration [3]. $\mathrm{CO}_{2}$ can remain in the atmosphere for almost 100 years, and thus, Earth's temperature will remain elevated in the coming decades. The risk for more severe changes to the climate and Earth's ecosystems will increase as the temperature of Earth's surface continues to rise. Our choices to reduce GHG emission today will shape the world in which the next generations will live. Thus, reductions in vehicle energy consumption and emissions are crucial. The U. S. Department of Transportation (USDOT) investments in the intelligent transportation system (ITS) platform will lead to a second revolution in the U. S. transportation industry. ITS confirmed the use of wireless communication technology in enhancing transportation system efficiency and reducing transportation's environmental impact. A new wave of environmentally aware transportation technology research targeting different areas of renewable energy sources and improved energy efficiency machinery has developed in the $\mathrm{U}$. S. The electric vehicle $(\mathrm{EV})$ is emerging as a state-of-the-art technology vehicle addressing the continually pressing energy and environmental concerns. Other proposed potential solutions include static- and dynamic-based vehicle routing advisory systems that would guide drivers through a minimized fuel consumption travel route, formerly recognized as "environmentally friendly routing/eco-routing".

The work in this paper is inspired by the USDOT's vision of the connected vehicle [4] and introduces a vehicle routing methodology that reduces the effect of traffic congestion by integrating a predictive mechanism for traffic congestion assessment and mitigation. This paper proposes an eco-predictive dynamic routing methodology advising the driver of the path with the least emissions based on a predictive (futuristic) traffic congestion assessment. Eco-predictive dynamic routing is evaluated with real-time dynamic routing, which dynamically advises the driver of the path with the least emissions based on real-time (current) traffic information. In addition, the evaluation is extended to include a conventional static routing methodology, which advises the driver of the path with the least emissions at the start of a trip based on historical traffic information. This paper identifies the significance of routing policies based on predictive traffic data and incorporates this information as a key factor in the routing policy. This paper's specific contribution to the knowledge base of vehicle routing methodologies in cost-dependent networks is summarized as follows:

1. Establish a novel framework for environmentally friendly routing in a dynamic cost-dependent network.

2. Extend the study of environmentally friendly routing policies to strategies based on predictive traffic information.

3. Validate our proposed methodology by developing a traffic simulation tool integrating the proposed Eco-Predictive Dynamic Routing methodology.
The paper is organized as follows: Section 2 discusses related work; the proposed system architecture and our assumptions are introduced in Section 3; the different routing methodologies are presented in Section 4; Section 5 presents the model and selection background; simulation results comparing the fuel consumption, emissions, and travel time of each trip are discussed in Section VI; finally, the conclusions are drawn in Section VII.

\section{Related Work}

Recent developments in vehicle electrification have led to significant improvements in vehicle operating efficiency. Overall, electric propulsion and charging efficiency offer nearly three-fold-higher efficiency (approximately 68\%) compared to conventional internal combustion propulsion vehicles (approximately 23\%). Electricity generation and production accounted for $40 \%$ of GHG emissions in 2011 [5, 6]. Whereas vehicle electrification offers a way to reduce vehicle emissions, it has shifted the emission generation from being on-board to off-board at the electric utility companies. Another field of vehicle efficiency research has proposed an optimal speed advising methodology to reduce vehicle fuel consumption and emissions [7]. The optimal speed advisory eco-driving system is rendered less practical in congested areas if not combined with an energy-efficient travel route selection. None of the existing eco-driving advisory systems optimize both the drive profile and route profile, which provides the true benefits of environmentally friendly transportation, as represented in Figure 1. Recent navigation system research has proposed an energy consumption/emission routing algorithm using historical traffic data modeling. More than $50 \%$ of congestion is non-recurring congestion caused by accidents (25\%), weather conditions $(15 \%)$, and temporary construction (10\%) [8]. Nonrecurring congestion reduces the availability of the traffic network, thus rendering a system based on historical traffic data insufficient in $50 \%$ of cases. Real-time traffic data modeling [9] provides enhanced performance in traffic congestion assessments; however, improved performance is expected with a predictive traffic congestion model with increased certainty. The assessment of predictive traffic information modeling using wireless communication data has been limited due to the difficulty in objectively and quantitatively evaluating energy and emission reduction effects using ITS.

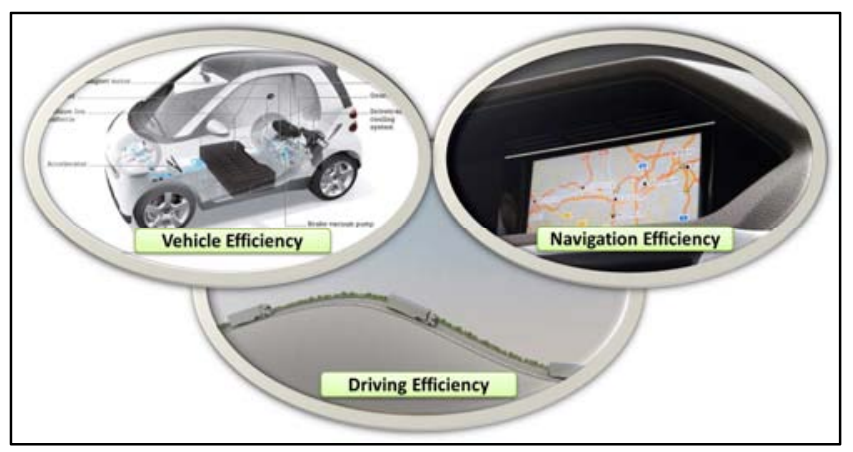

Figure 1. Environmentally friendly transportation strategies. 
The Intelligent Transportation Society of America (ITS America) has invested and continues to invest in the development and deployment of ITS services relying on the wireless communication technology formerly known as DSRC. Vehicles equipped with DSRC technology offer a promising opportunity for energy and emission optimization navigation applications and are the original intent of the DSRC $5.9 \mathrm{GHz}$ frequency band allocation by the Federal Communications Commission (FCC) for vehicle safety applications. There are several vehicle routing methodologies offering static shortest distance and time routing [9-11]. However, none of the methodologies proposed thus far have been identified in the field of eco-predictive dynamic navigation. The literature survey revealed several vehicle onboard routing algorithms employing simplified modeling techniques that may not be suitable for providing a true energy/emission-optimized route. The existing routing system does not use dynamic routing to allow drivers to avoid abrupt traffic changes, such as traffic accidents. Furthermore, the current routing algorithm considers real-time traffic information but not predictive traffic information. Because the traffic condition is relevant at the navigated vehicle's expected time of arrival, predictive traffic information should improve route performance in terms of travel time, energy consumption, and emissions. To overcome the shortcomings of existing environmentally aware navigation systems, our proposed methodology provides a predictive traffic information assessment and integration methodology to dynamically find an energy- and emission-efficient route.
Furthermore, vehicle on-board navigation systems have limited computational power and need not be overburdened with additional computational functions. Although the proposed on-board algorithm will be optimized for computational time efficiency, an off-board traffic data mining, dissemination, and classification architecture is proposed to further alleviate the on-board system from additional calculation functions.

\section{Proposed System Architecture}

Environmentally friendly routing is a methodology intended to enable reductions in energy consumption and emissions to reduce global warming implications. The shortest or fastest path does not necessarily provide emission-optimized routing in all traffic conditions; vehicle energy consumption and emissions depend heavily on changing traffic conditions. A highly congested but quick route does not offer minimum travel time or emissions. An increased number of vehicles on a limited roadway yields decreased route throughput and results in longer vehicle waiting time, formerly known as congestion. Similarly, a congested but short path offers the minimum travel distance but does not minimize travel time, energy consumption, or emissions. An emission-optimized route that incorporates traffic, vehicle, and geographical information can allow the vehicle to move from the origin to the destination with the shortest travel time and least emissions. The three main routing objectives are illustrated in Figure 2.

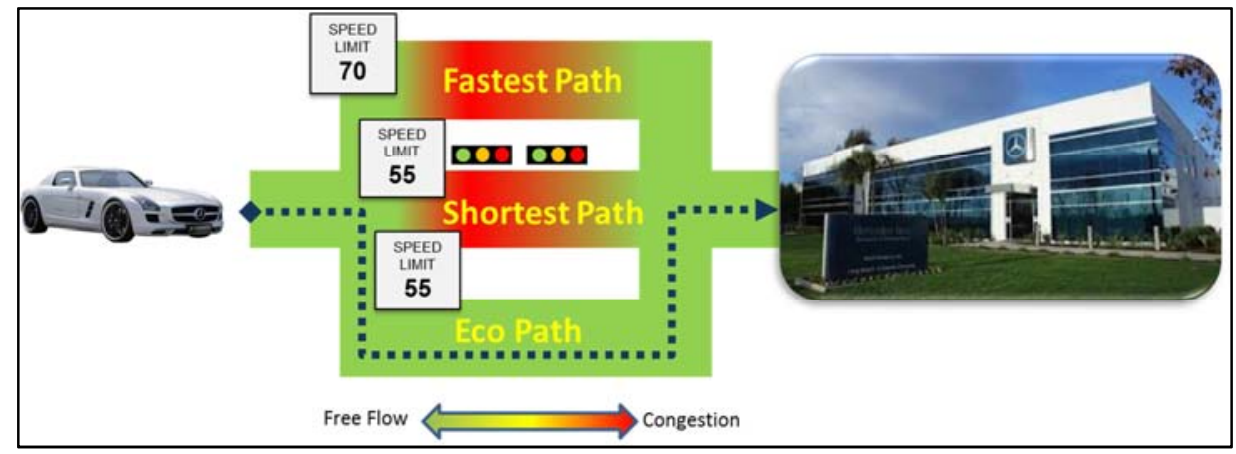

Figure 2. Vehicle routing objectives.

1. The optimized navigation is achieved via wireless communication of traffic information using vehicle-to-vehicle (V2V), vehicle-to-infrastructure (V2I), and infrastructure-to-vehicle (I2V) platforms and on-board vehicle travel route optimized applications. The overall methodology for developing eco-friendly navigation can be constructed in three phases, as depicted in Figure 3 and described below:

2. Phase I is the initialization step, where the roadway network covering the origin to the destination is partitioned and represented as a number of nodes and segments. This step uses vehicle and route parameters at the origin to advise the driver for initial drive and route profiles based on real-time traffic data.

3. Phase II uses the driver input to develop the travel cost function to be optimized. For a given traffic network, segment-based travel cost factors are developed based on the traffic and roadway conditions. The segment-based factors are based on the microscopic segment characteristics, such as vehicle travel profile (speed vs. time), route profile (location vs. time), and road profile (road gradient). The segment-based factors are indexed in a Predictive Traffic Congestion Index (PTCI).

4. Phase III uses travel time cost parameters to plan an optimized route and drive profiles. Provided the PTCI, a routing policy and novel dynamic re-routing policy based on predictive traffic information are developed to enhance vehicle performance relative to emissions and travel time. The proposed method illustrates that a stochastic model does not need to be included if vehicles 
adhere to the drive/route profiles and the traffic information is dynamically updated.

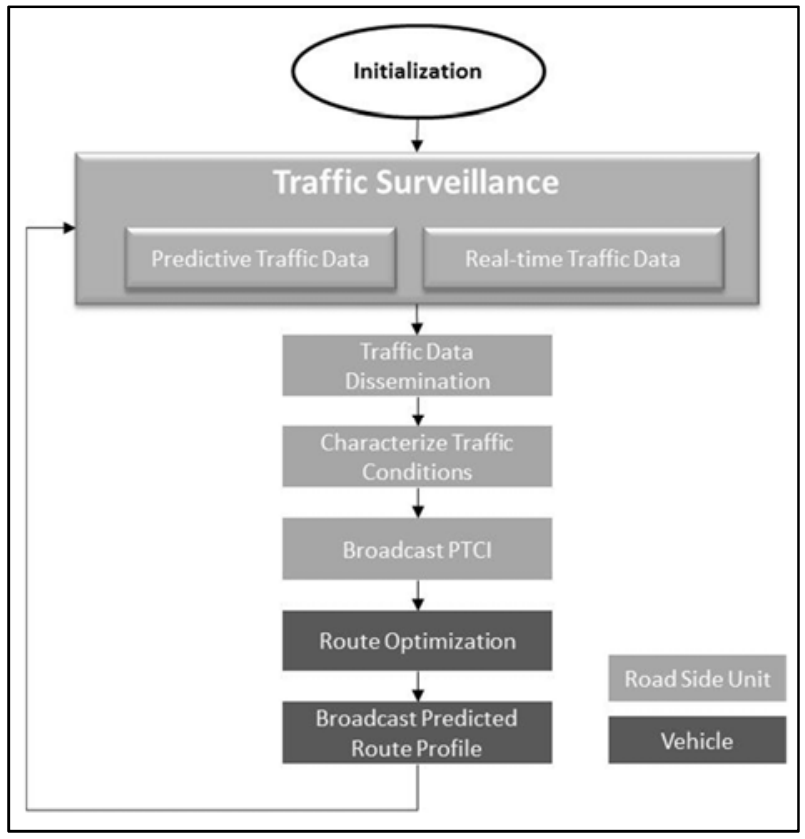

Figure 3. Eco-friendly navigation flow diagram.

\section{Routing Methodology}

Finding the optimal route in a road network from a current start location to a given destination is an everyday problem that most drivers must consider when planning a trip. The term "optimal" in a routing algorithm may refer to a range of objectives from which end-users can choose to optimize the route, such as the fastest route, shortest route, fastest route given a preference to various road characteristics, or most fuel-efficient route. These navigation algorithms also differ in the way that they address the changing traffic conditions over time, and they can be divided into the two main categories shown in Figure 4, namely, static and dynamic.

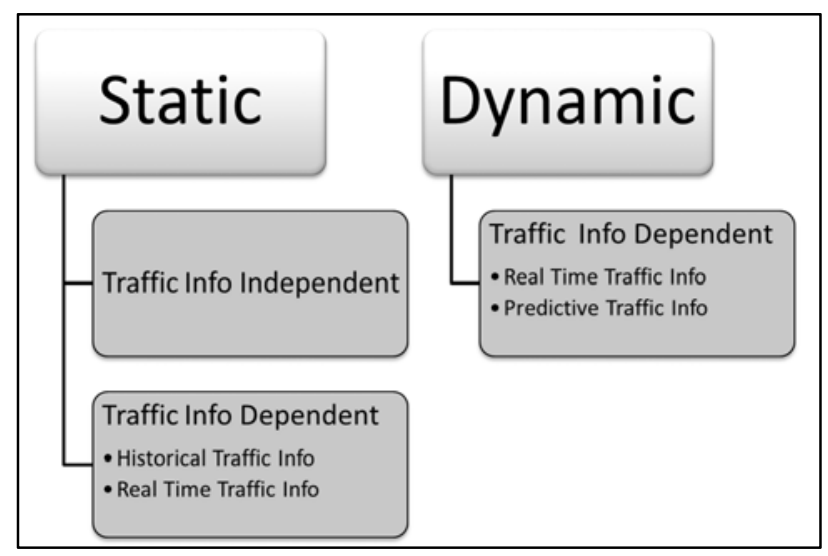

Figure 4. Routing computation methods.

In the conventional static planning model, all travel times and traffic conditions are considered constant over time, resulting in less realistic travel costs for road segments. The static model was improved upon in the extended deterministic static model [12], in which certain properties of the traffic network are considered to change as a function of the time of day, week, or even season (e. g., some roads may be closed during specific time periods); thus, the route cost estimation is more accurate. Most commercial navigation systems use the extended deterministic planning model. However, a more accurate representation of the traffic flow and responsive routing can be achieved with a dynamic routing model [13].

A. Traffic Information Assessment and Communication

Communication of real-time traffic conditions between vehicles and infrastructures is an essential functional requirement to achieve a predictive vehicle navigation system with enhanced traffic assessment accuracy. The dynamic nature of traffic conditions requires the employment of low-latency communication technology. DSRC was primarily developed for ITS vehicular safety applications to support a vehicle traveling at high speed to communicate status in $\mathrm{V} 2 \mathrm{~V}$, V2I, and I2V configurations. DSRC offers the highest messaging latency performance compared to the proposed alternative communication technologies, including Fourth-Generation Long-Term Evolution (4G LTE), its successor (5G LTE), and Wi-Fi, as summarized in Table 1. Furthermore, DSRC communication allows for essential traffic congestion assessment with a predictive modeling capability feature with no separate infrastructure, such as the previously proposed approaches listed below and limited to real-time traffic information assessment:

i. Optical Digital Camera [14]: this methodology is based on analyzing consecutive video images of a specific road network segment.

ii. Inductive Loop Road Sensor [15]: this methodology employs the use of magnetic sensors embedded in the road to count the number of vehicles passing through a road segment.

Table 1. Comparison of wireless communication technologies.

\begin{tabular}{lllll}
\hline & $\begin{array}{l}\text { DSRC } \\
(\mathbf{8 0 2 . 1 1})\end{array}$ & $\begin{array}{l}\text { Wi-Fi } \\
(\mathbf{8 0 2 . 1 1 n})\end{array}$ & 4G LTE & 5G \\
\hline Frequency & $5.8 \mathrm{GHz}$ & $5.2 \mathrm{GHz}$ & $2 \mathrm{GHz}$ & $28 \mathrm{GHz}$ \\
Bandwidth & $75 \mathrm{MHz}$ & $20 \mathrm{MHz}$ & $20 \mathrm{MHz}$ & Not defined \\
Range & $<1000 \mathrm{~m}$ & $<250 \mathrm{~m}$ & $<1000 \mathrm{~m}$ & $<2000 \mathrm{~m}$ \\
Data rate & $<27 \mathrm{Mbps}$ & $<54 \mathrm{Mbps}$ & $<75 \mathrm{Mbps}$ & $<1 \mathrm{Gbps}$ \\
Latency & $<50 \mathrm{~ms}$ & $<100 \mathrm{~ms}$ & $<100 \mathrm{~ms}$ & $<1 \mathrm{~ms}$ \\
Mobility & $>60 \mathrm{mph}$ & $>60 \mathrm{mph}$ & $>60 \mathrm{mph}$ & $>60 \mathrm{mph}$ \\
V2V & Yes & Yes & Thru Server & Thru Server \\
V2I & Yes & Yes & Yes & Yes \\
I2V & Yes & Yes & Yes & Yes \\
\hline
\end{tabular}

\section{B. Predictive Traffic Information}

Our methodology to model and estimate traffic conditions is based on the extensively used Greenshield model [16]. The model considers a linear relationship between the traveled vehicle speed and congestion level, as illustrated in Figure 5. Applying the proposed predictive and dynamic route search algorithm requires iterative calculations to find an optimal solution. This calculation becomes computationally intensive when considering a large number of vehicles and route options, resulting in an exponentially long computation time. 
This phenomenon is known as the "Curse of Dimensionality" proposed by Richard Bellman [17] in reference to the optimization by exhaustive enumeration of an open-ended search space. To mitigate this phenomenon, it is important to develop and integrate a functional approximation architecture to represent the travel cost. The approximation architecture of current and predictive traffic information is a key property for high precision in traffic condition assessment. For every roadway segment, link-based travel cost factors have been developed based on the traffic conditions. Henceforth, the link-based weight is referenced as the PTCI, which is calculated based on real-time and predictive traffic conditions. The PTCI consisting of travel cost is computed in the road-side unit (RSU) by assessing the vehicle travel cost employing the V2I and I2V communication infrastructure and given the distance of each road segment as logged. Each route segment has an equilibrium point at which free flow occurs at the vehicle speed limit, i. e., the number of vehicles arriving to the segment equals the number of vehicles leaving the segment, as illustrated in Figure 5.

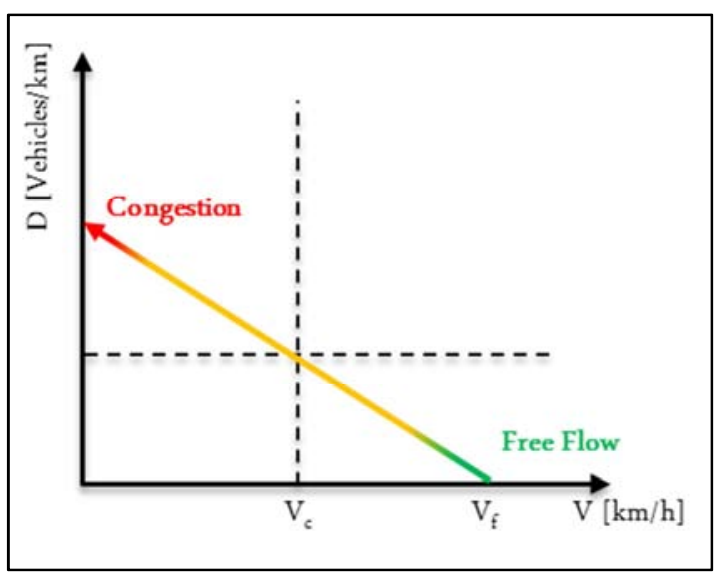

Figure 5. Traffic density vs. vehicle speed.

1. The PTCI concept is introduced to represent vehicle flow and density with an associated level of congestion and is stored in the RSU for reference by future non-navigated vehicles. This section describes the methodology for calculating the PTCI. The traffic characteristics used in the calculation of the PTCI illustrated in Figure 6 are described below.

2. Headway $(\mathrm{H})$ : time between two successive vehicles (s/vehicle)

3. Gap $(G)$ : time between two successive vehicles excluding the length of the leading vehicle ( $\mathrm{s} /$ vehicle)

4. Inflow $\left(Q_{\text {pin }}\right)$ : number of vehicles predicted to pass the starting point in a new segment during a specified time interval (vehicles/h)

5. Outflow $\left(Q_{\text {pout }}\right)$ : number of vehicles passing the end point in a new segment during a specified time interval (vehicles/h)

6. V: vehicle speed $(\mathrm{km} / \mathrm{h})$

7. $\mathrm{V}_{\mathrm{f}}$ : free-flow vehicle speed $(\mathrm{km} / \mathrm{h})$

8. $\mathrm{V}_{\mathrm{p}}$ : predictive speed $(\mathrm{km} / \mathrm{h})$

9. $\mathrm{D}_{\mathrm{p}}$ : predictive traffic density, number of vehicles occupying segment space (vehicles $/ \mathrm{km}$ )

10. $\mathrm{D}_{\mathrm{j}}$ : traffic jam density (vehicles $/ \mathrm{km}$ )

11. The inverse of flow is headway $(\mathrm{H})$, which is the time between the $i^{\text {th }}$ vehicle entering segment and the $(i+1)^{\text {th }}$ vehicle

12. $\mathrm{T}_{\mathrm{p}}$ : predictive travel time $(\mathrm{s})$

13. $\mathrm{L}_{\mathrm{i}}$ : travel segment length $(\mathrm{m})$

14.The traffic model considers a linear relationship between the vehicle speed, traffic density, and flow; thus:

$$
\begin{gathered}
V_{p}=V_{f}\left(1-\frac{D_{p}}{D_{j}}\right) \\
V_{p}=\frac{Q_{p}}{D_{p}} \\
T_{p}=\frac{L_{p}}{V_{p}}
\end{gathered}
$$

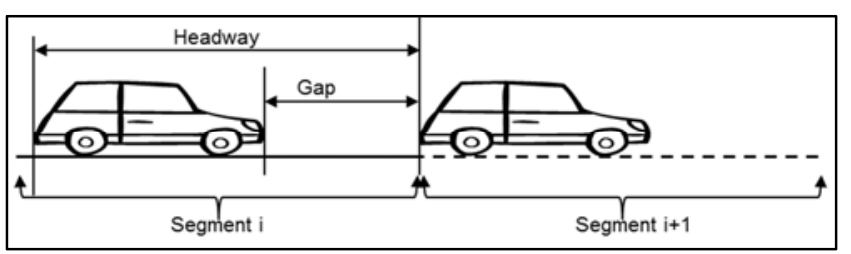

Figure 6. Traffic characteristics.

Table 2 illustrates the PTCI for a single-lane scenario in which $\mathrm{V}_{\mathrm{f}}=50 \mathrm{~km} / \mathrm{h}$, and $\mathrm{L}_{\mathrm{i}}=400 \mathrm{~m}$. Under the aforementioned conditions, travel time costs are calculated for the respective segment based on equation (3), and a corresponding PTCI is selected and updated in the RSU to be communicated to the navigated vehicle for a decision on re-routing.

Table 2. Travel time cost scenario.

\begin{tabular}{lll}
\hline Travel Time (s) & PTCI & Description \\
\hline $288-308$ & 0 & Free Flow \\
$309-329$ & 1 & Slight Delay \\
$330-350$ & 2 & Moderate Delay \\
$351-371$ & 3 & High Delay \\
$372-392$ & 4 & Significant Congestion \\
$>392$ & 5 & Severe Congestion \\
\hline
\end{tabular}

Similarly, the predicted emission $\delta_{\mathrm{p}}$ can be calculated based on the Handbook of Emission Factors (HBEFA) for the road transport emission model [18], as defined in the emission fit function [19] depicted below:

$$
\begin{gathered}
\delta_{p}(v, a)=\frac{1}{h \gamma}\left(P_{0}+m \cdot a_{p} \cdot v_{p}+m \cdot g \cdot \mu_{0} \cdot v_{p}+m \cdot g \cdot \beta \cdot v_{p}+\right. \\
\left.m \cdot g \cdot \mu_{1} \cdot v_{p}{ }^{2}+\frac{1}{2} c_{w} \rho A v_{p}{ }^{3}\right)
\end{gathered}
$$

where $\mathrm{c}_{\mathrm{w}}$ is the aerodynamic resistance, $\mathrm{m}$ is the vehicle mass, $\mu_{0}$ is the static friction coefficient, $\mu_{1}$ is the dynamic friction coefficient, $\mathrm{P}_{0}$ is the idle power consumption, $\gamma$ is the motor efficiency, $\mathrm{A}$ is the front area of the vehicle, $\mathrm{h}$ is the energy content, $\rho$ is the air density, $g$ is the gravitational constant, and $\beta$ is the road segment slope.

Table 3 illustrates the PTCI for a single-lane scenario with 
$\mathrm{V}_{\mathrm{f}}=50 \mathrm{~km} / \mathrm{h}, \mathrm{L}_{\mathrm{i}}=400 \mathrm{~m}$, and a road segment slope of $0 \%$. Under the aforementioned conditions, travel costs are calculated for a respective segment based on equation (4), and a corresponding PTCI is selected and updated in the RSU to be communicated to the navigated vehicle for a decision on re-routing. One vehicle class weight is considered here; however, the vehicle model could be extended in future work to incorporate additional vehicle classes, such as light-, medium-, and heavy-duty vehicles.

Table 3. Emission travel cost scenario.

\begin{tabular}{lll}
\hline $\mathbf{C O}_{2}$ Emission (grams) & PTCI & Description \\
\hline$<165$ & 0 & Free Flow \\
$166-180$ & 1 & Slight Delay \\
$181-205$ & 2 & Moderate Delay \\
$206-220$ & 3 & High Delay \\
$221-235$ & 4 & Significant Congestion \\
$>235$ & 5 & Severe Congestion \\
\hline
\end{tabular}

\section{Dynamic Re-routing}

During travel, an alternative route search is performed if traffic congestion is increased, which is reflected in a higher PTCI value. In the proposed dynamic routing methodology, predictive traffic information is integrated into the planning model. The optimal route to the destination is calculated before the start of a trip and is updated dynamically to adapt to new traffic conditions during travel, as illustrated in Figure 7. When multiple travelers make dynamic routing decisions to the same alternative route, special consideration should be given to prevent vehicles from taking the same alternative path during a traffic jam. The proposed dynamic re-routing logic is activated when the updated received PTCI is worse, reflecting worsening traffic conditions $\left(\mathrm{PTCI}_{\text {new }}>\right.$ $\mathrm{PTCI}_{\text {previous }}$ ). It is important to select the proper PTCI threshold for the re-routing decision to avoid unnecessary or delayed re-routing. Furthermore, the number of re-routing events was limited to two to alleviate the computational burden. The vehicles selected for re-routing are the vehicles closest to the identified predictive traffic congestion. The distance factor is integrated in the on-board vehicle application and is assumed to be the same for all other vehicles. The union of all vehicles closest to the predicted congestion is selected and evaluated for alternative constrained paths.

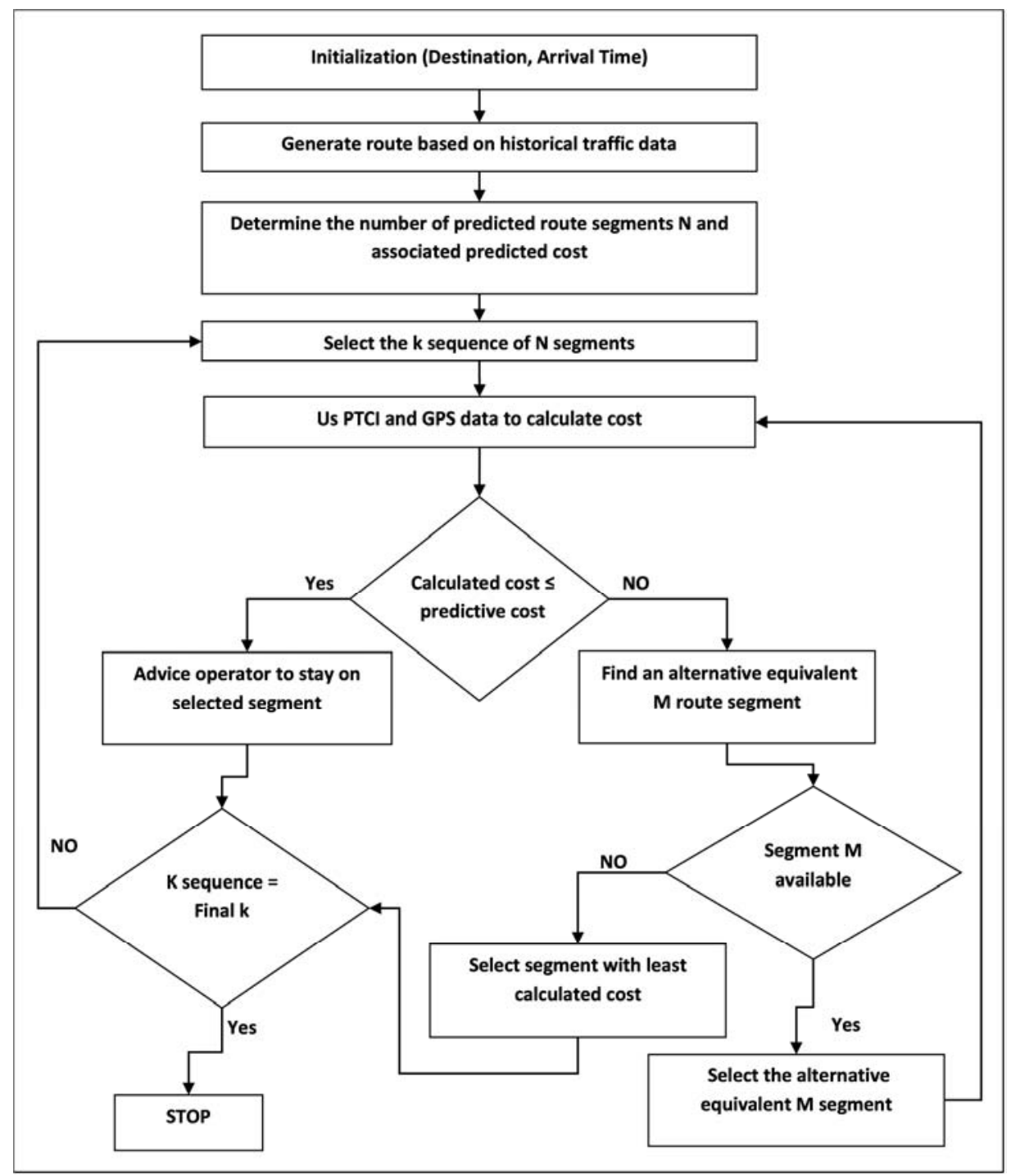

Figure 7. Operational flow chart diagram. 


\section{Traffic Network Model}

The transportation system is a rather complex, asynchronous, and dynamic system. Its structure is characterized as a discrete event dynamic system (DEDS) [20] in which state transitions are triggered by the occurrence of discrete physical events that occur randomly in the system; thus, the traffic system can be modeled by Petri nets (PNs). The capability of PNs extends beyond other similar mathematical modeling languages (e. g., neural networks) to include analysis, control, and graphical representation. Since their introduction in 1962 by Carl Adam Petri, PNs have been extensively applied to transportation systems. A literature survey documenting all PN applications in the modeling, analysis, and control of traffic systems has been presented, indicating the potential extension of PNs to transportation systems [21]. The navigation process of a vehicle can be approached as a discontinuous system of connected road segments. PNs offer a graphical representation of the system that consists of places, transitions, arcs, and tokens. In the transportation model, places are represented by interconnecting points that enable re-routing, transitions represent travel costs, arcs represent paths between RSUs, and tokens are vehicle-constrained travel parameters (e. g., travel time or emissions). In the simplified traffic network and equivalent PN model scenario illustrated in Figure 8, it is necessary to unfold the PN to analyze the reachability of the destination by the vehicle at the origin. The unfolding approach of $\mathrm{PN}=(\mathrm{P}, \mathrm{T}, \mathrm{F}$, $\left.\mathrm{C}_{0}\right)$ is $\Omega=(\mathrm{RN}, \beta)$, where $\mathrm{RN}$ is a resulting net, $\beta$ is the monomorphic category of net $\mathrm{N}$ to $\mathrm{RN}$, and conditions $\mathrm{C}$ and events $\mathrm{E}$ are mapped to place $\mathrm{P}$ through transition $\mathrm{T}$.

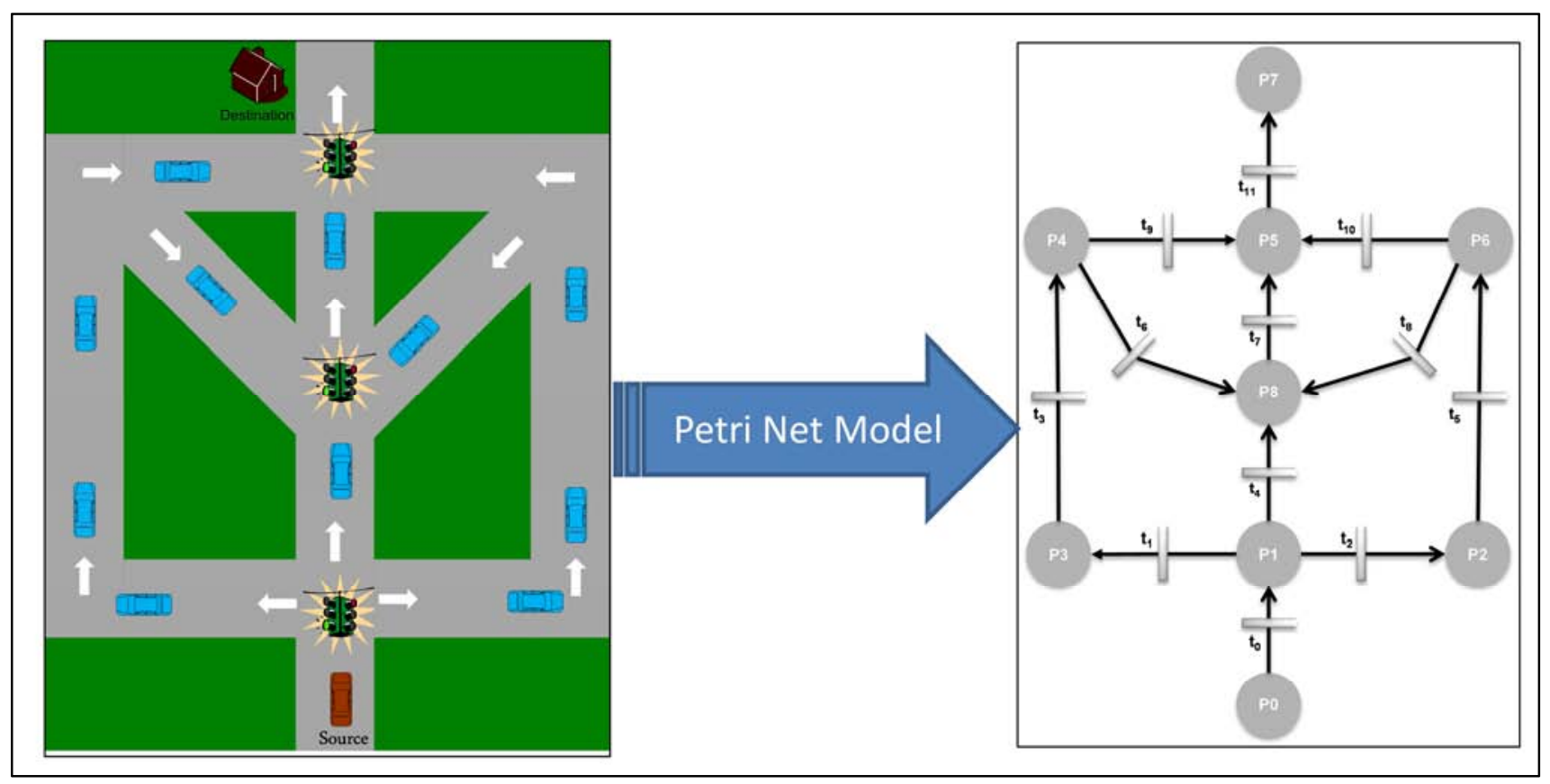

Figure 8. Traffic network and equivalent $P N$ model.

The PN unfolding process first introduced by McMillan $[22]$ is a reachability analysis and planning tool that naturally enables the identification of all possible paths from an origin to destination and allows for the determination of separate solutions for independent sub-problems. The reachability algorithm involves a search strategy but does not require a specific methodology. Typically, a breadth-first search (BFS) algorithm is implemented. In this paper, we implemented a modified Dijkstra's algorithm [23] that we have named the arc cost compliment (ACC) algorithm. Provided that route planning is properly coupled to the algorithm, the ACC algorithm generates an optimal navigation relative to travel time. The forthcoming section provides the definition and notation used in the algorithm and presents the details of the ACC algorithm.

A. Definition

The objective of environmental navigation is to move a vehicle from an origin to a destination while avoiding traffic congestion and optimizing travel time. The problem space can be formulated as a set of places denoting vehicle positions, destinations, and roadway intersections connected by unidirectional arcs representing connecting paths, each of which has an associated travel cost. The vehicle starts at the origin and moves across arcs to other states until it reaches the destination state. The travel cost for traversing an arc from place $\mathrm{P}$ to $\mathrm{P}_{\mathrm{i}}$ is the positive compliment number of the arc travel cost. If $\mathrm{P}$ does not have an arc to $\mathrm{P}_{\mathrm{i}}$, then $\mathrm{c}\left(\mathrm{P}, \mathrm{P}_{\mathrm{i}}\right)$ is undefined. Two places, $\mathrm{P}$ and $\mathrm{P}_{\mathrm{i}}$, are connected in space if $\mathrm{c}$ $\left(\mathrm{P}, \mathrm{P}_{\mathrm{i}}\right)$ is defined. The routing problem to minimize the sum of the total travel time is formulated as an optimization algorithm. The place $p_{v i, u j}$ is introduced to denote the existence of a vehicle $v_{i}$ on roadside unit $u_{j}$. The firing of each transition $t_{v i, u a, u b}$ implies the traveling of a vehicle between two adjacent RSUs, $\mathrm{u}_{\mathrm{a}}$ and $\mathrm{u}_{\mathrm{b}}$. Let $\mathrm{N}_{\mathrm{t}}$ be the total number of possible paths to 
be evaluated for vehicle $v_{i}$. Let $M_{k}$ be the initial marking at time k. Let $\varphi_{\text {ui }}$ be the total travel time threshold and $\delta_{u i}$ be the total emission. The routing problem can then be formulated as the problem of finding an optimal firing sequence to minimize

$$
\mathrm{C}=\sum_{p=}^{P i} \varphi_{u i}+\delta_{u i}
$$

\section{B. Optimization Algorithm}

Step 1: Initialization

a) The number of travel time constraints is set as the number of tokens at $\mathrm{P}_{0}$

b) The pre-calculated travel time cost is set as the arc weight

c) The pre-calculated emission cost is set as the arc weight

d) Starting at the initial origin node, search the markers that enable a transition

Step 2: Optimization

a) Fire a transition and then decrease the number of tokens from its initial marker

b) Continue firing until the number of tokens is depleted or destination $P_{i}$ is reached

c) Repeat for all possible paths $\mathrm{N}_{t}$ and save all possible interim solutions

Step 3: Convergence

Based on the driver's routing selection for travel time or emission, the optimal solution is the path connecting the origin to the destination with a maximum number of tokens remaining at the destination.

Step 4: Re-Optimization

The re-optimization of subnet $u_{j}$ is executed on the condition that higher arc weights are received, indicating worsening traffic conditions. The objective function for re-optimization is similar to step 2 , given that re-optimization begins at the current marker.

C. Finite Converged Search

An infinite unfolding of PN $\Omega$ will result from a transportation network in which all route segments are connected. Thus, we seek a complete finite unfolded alternative $\Omega$ ' containing a sufficient amount of information to reach an optimal solution. The key to obtain a complete, finite prefix is to identify the unfolding cut-off event, which is the node connection capacity in our case. The node connection capacity is selected as the cut-off event, as the driver in a dynamic routing environment is capable of switching to alternative routes when a route has been taken with multiple node connections. The node capacity $(\sigma)$ is defined as the number of segments available from any node $\mathrm{P}_{\mathrm{i}}$, representing all possible routes for an origin-destination pair. The node capacity of a route is built when the origin node $P_{i}$ starts to identify a path to the destination node $P_{d}$. The maximal node capacity of node $\mathrm{P}_{\mathrm{i}}$, denoted as $\sigma_{\mathrm{i}}$, indicates the available connecting segments at the subsequent node, $\mathrm{P}_{\mathrm{i}+1}$. The node capacity is a good measure for the node degree of travel freedom, providing a reliability assessment of the path. The node capacity serves as an essential tool in route planning for determining how to select the path to the destination efficiently while ensuring that the driver will arrive at the destination at any cost in the case of a link connection failure (due to, for example, an accident). The node capacity is determined for each node in the roadway network; the node capacity for node $\mathrm{P}_{\mathrm{i}+1}$ is attached to the previous connecting node or nodes $\mathrm{P}_{\mathrm{i}}$. The reason for this result is that when a driver is traveling a segment, he cannot reroute until the next node is reached. In cases where U-turns are allowed in a roadway network, those U-turns are to be considered as separate nodes. U-turns are not reflected in this example and will be addressed in future work. The unfolding action is ceased when the defined node capacity $\sigma$ ' is reached. Figure 9 and Figure 10 present the unfolding of nodes P5 and P8, respectively, to evaluate the reachability to the final marker P7.

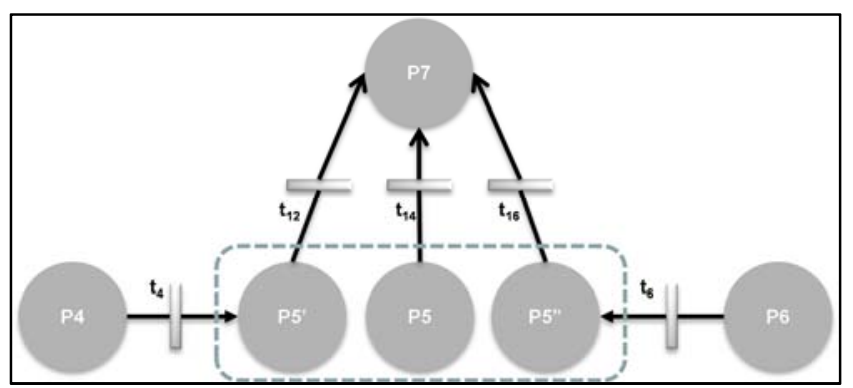

Figure 9. Unfolding P5 through P4 and P6.

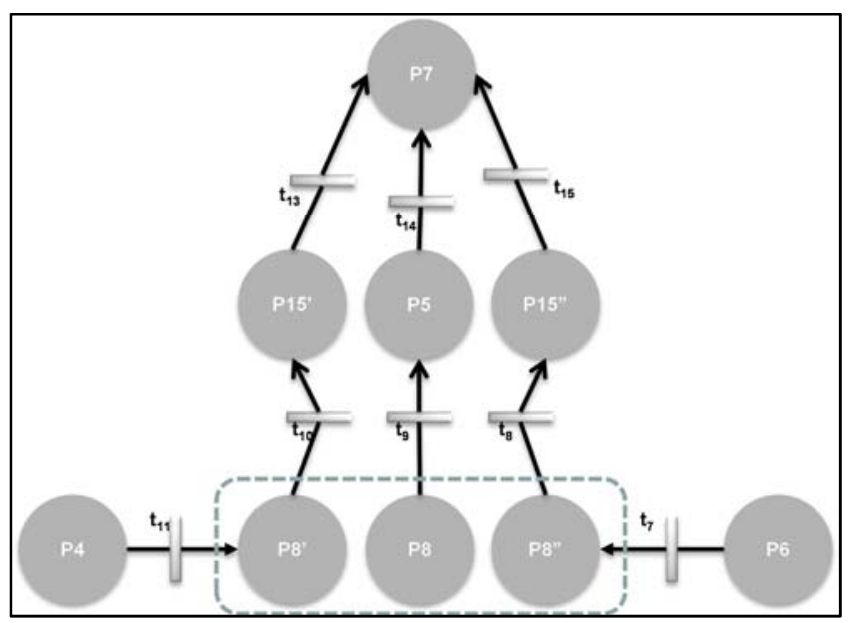

Figure 10. Unfolding: P8 through P4 and P6.

Figure 11 presents the fully unfolded PN corresponding to the traffic network given in Figure 8. All possible paths from the origin to destination are represented. Because the travel time and emissions are the target optimized travel costs, the initial marking $\mathrm{M}_{0}$ represents the upper bound travel time and emission constraints set. In this case, the constraints are $60 \mathrm{~s}$ and $200 \mathrm{~g}$ of $\mathrm{CO}_{2}$ based on real-time traffic data. The marker $\mathrm{M}_{\mathrm{ij}}$ is the final marker denoting the predictive travel time and emission cost compliment for the respective path $\mathrm{j}$. 


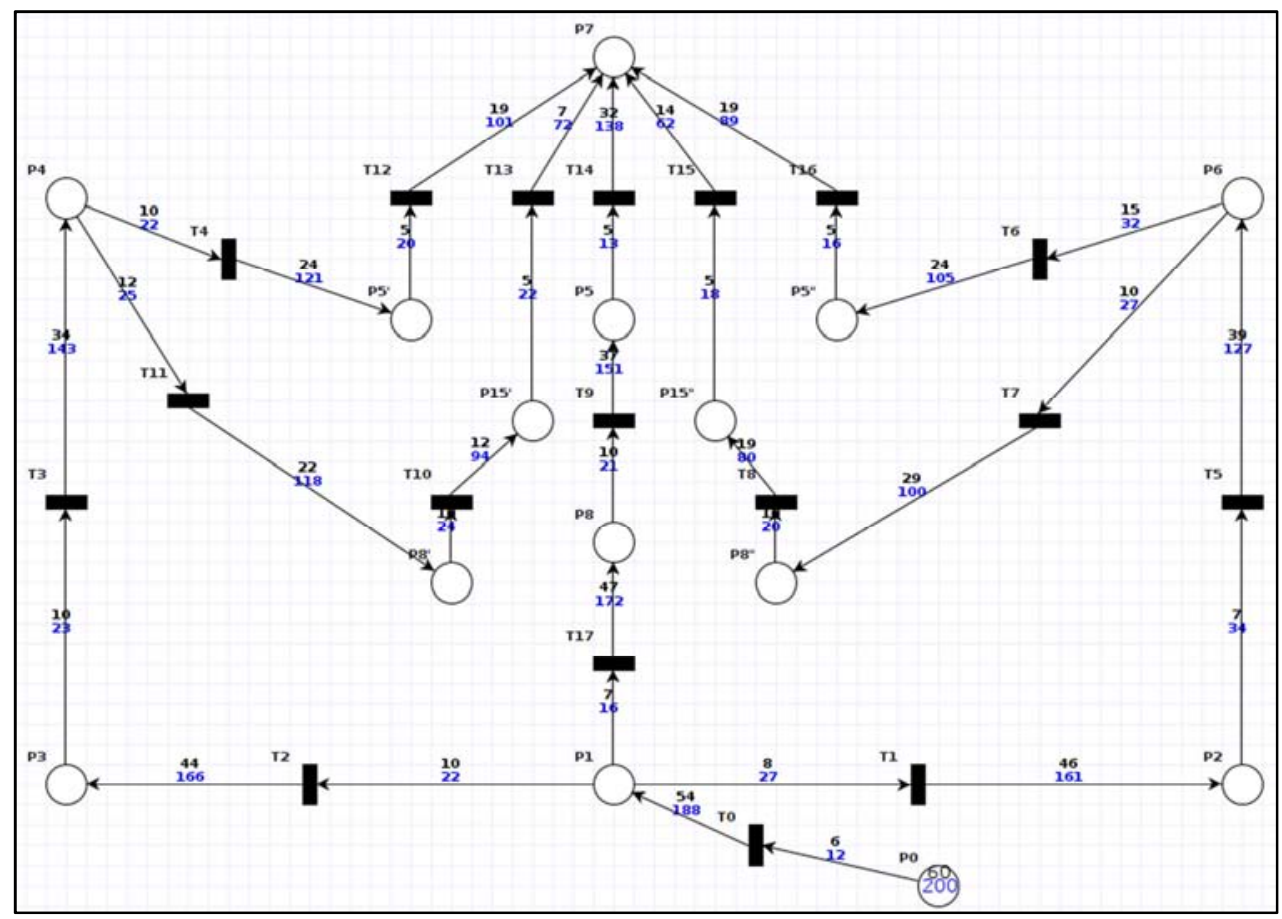

Figure 11. Unfolded PN model.

The vehicle destination P7 can be reached via different places and firing sequences. The incidence matrix depicted in Figure 12 presents all possible paths and presents the travel time cost compliment of each path based on the ACC algorithm. The final destination $\mathrm{P} 7$ can be reached via transition $T_{i}$ and respective cost $C_{i}$ as T12:41, T13:53, T14:28, $\mathrm{T} 15: 46$, or $\mathrm{T} 16: 41$. The lowest-cost path through transitions $\mathrm{T} 0 \rightarrow \mathrm{T} 17 \rightarrow \mathrm{T} 9 \rightarrow \mathrm{T} 14$ is selected as the optimal route plan.

\begin{tabular}{|l|c|c|c|c|c|c|c|c|c|c|c|c|c|c|c|c|c|c|}
\hline & T0 & T1 & T10 & T11 & T12 & T13 & T14 & T15 & T16 & T17 & T2 & T3 & T4 & T5 & T6 & T7 & T8 & T9 \\
P1_Time & 54 & 0 & 0 & 0 & 0 & 0 & 0 & 0 & 0 & 0 & 0 & 0 & 0 & 0 & 0 & 0 & 0 & 0 \\
P0_Time & 0 & 0 & 0 & 0 & 0 & 0 & 0 & 0 & 0 & 0 & 0 & 0 & 0 & 0 & 0 & 0 & 0 & 0 \\
P2_Time & 0 & 46 & 0 & 0 & 0 & 0 & 0 & 0 & 0 & 0 & 0 & 0 & 0 & 0 & 0 & 0 & 0 & 0 \\
P15'_Time & 0 & 0 & 12 & 0 & 0 & 0 & 0 & 0 & 0 & 0 & 0 & 0 & 0 & 0 & 0 & 0 & 0 & 0 \\
P8'_Time & 0 & 0 & 0 & 22 & 0 & 0 & 0 & 0 & 0 & 0 & 0 & 0 & 0 & 0 & 0 & 0 & 0 & 0 \\
P4_Time & 0 & 0 & 0 & 0 & 0 & 0 & 0 & 0 & 0 & 0 & 0 & 34 & 0 & 0 & 0 & 0 & 0 & 0 \\
P7_Time & 0 & 0 & 0 & 0 & 19 & 7 & 32 & 14 & 19 & 0 & 0 & 0 & 0 & 0 & 0 & 0 & 0 & 0 \\
P5'_Time & 0 & 0 & 0 & 0 & 0 & 0 & 0 & 0 & 0 & 0 & 0 & 0 & 24 & 0 & 0 & 0 & 0 & 0 \\
P5_Time & 0 & 0 & 0 & 0 & 0 & 0 & 0 & 0 & 0 & 0 & 0 & 0 & 0 & 0 & 0 & 0 & 0 & 37 \\
P15"_Time & 0 & 0 & 0 & 0 & 0 & 0 & 0 & 0 & 0 & 0 & 0 & 0 & 0 & 0 & 0 & 0 & 19 & 0 \\
P5"_Time & 0 & 0 & 0 & 0 & 0 & 0 & 0 & 0 & 0 & 0 & 0 & 0 & 0 & 0 & 24 & 0 & 0 & 0 \\
P8_Time & 0 & 0 & 0 & 0 & 0 & 0 & 0 & 0 & 0 & 47 & 0 & 0 & 0 & 0 & 0 & 0 & 0 & 0 \\
P3_Time & 0 & 0 & 0 & 0 & 0 & 0 & 0 & 0 & 0 & 0 & 44 & 0 & 0 & 0 & 0 & 0 & 0 & 0 \\
P6_Time & 0 & 0 & 0 & 0 & 0 & 0 & 0 & 0 & 0 & 0 & 0 & 0 & 0 & 39 & 0 & 0 & 0 & 0 \\
P8"_Time & 0 & 0 & 0 & 0 & 0 & 0 & 0 & 0 & 0 & 0 & 0 & 0 & 0 & 0 & 0 & 29 & 0 & 0 \\
\hline
\end{tabular}

Figure 12. Travel time cost incidence matrix.

If the driver selects the emissions-based route optimization search, the reachability to the destination is calculated in a similar manner. The vehicle destination place P7 can be reached via different places and firing sequences. The incidence matrix depicted in Figure 13 presents all possible paths and presents the travel emission cost compliment of each path based on the ACC algorithm. The final destination P7 can be reached via transition $T_{i}$ and respective cost $C_{i}$ as $\mathrm{T} 12: 101, \mathrm{~T} 13: 72$, T14:138, $\mathrm{T} 15: 62$, or $\mathrm{T} 16: 89$. The lowest-cost path through transitions $\mathrm{T} 0 \rightarrow \mathrm{T} 17 \rightarrow \mathrm{T} 9 \rightarrow \mathrm{T} 14$ is selected as the optimal route plan. This specific scenario, where the road slope is $0 \%$, illustrates that the 
emissions-based PTCI provides the same route path solution as the travel time-based PTCI solution.

\begin{tabular}{|l|c|c|c|c|c|c|c|c|c|c|c|c|c|c|c|c|c|c|c|}
\hline & $\mathrm{T} 0$ & $\mathrm{~T} 1$ & $\mathrm{~T} 10$ & $\mathrm{~T} 11$ & $\mathrm{~T} 12$ & $\mathrm{~T} 13$ & $\mathrm{~T} 14$ & $\mathrm{~T} 15$ & $\mathrm{~T} 16$ & $\mathrm{~T} 17$ & $\mathrm{~T} 2$ & $\mathrm{~T} 3$ & $\mathrm{~T} 4$ & $\mathrm{~T} 5$ & $\mathrm{~T} 6$ & $\mathrm{~T} 7$ & $\mathrm{~T} 8$ & $\mathrm{~T} 9$ \\
P1_Emisison & 188 & 0 & 0 & 0 & 0 & 0 & 0 & 0 & 0 & 0 & 0 & 0 & 0 & 0 & 0 & 0 & 0 & 0 \\
P0_Emisison & 0 & 0 & 0 & 0 & 0 & 0 & 0 & 0 & 0 & 0 & 0 & 0 & 0 & 0 & 0 & 0 & 0 & 0 \\
P2_Emisison & 0 & 161 & 0 & 0 & 0 & 0 & 0 & 0 & 0 & 0 & 0 & 0 & 0 & 0 & 0 & 0 & 0 & 0 \\
P15'_Emisison & 0 & 0 & 94 & 0 & 0 & 0 & 0 & 0 & 0 & 0 & 0 & 0 & 0 & 0 & 0 & 0 & 0 & 0 \\
P8'EEmisison & 0 & 0 & 0 & 118 & 0 & 0 & 0 & 0 & 0 & 0 & 0 & 0 & 0 & 0 & 0 & 0 & 0 & 0 \\
P4_Emisison & 0 & 0 & 0 & 0 & 0 & 0 & 0 & 0 & 0 & 0 & 0 & 143 & 0 & 0 & 0 & 0 & 0 & 0 \\
P7_Emisison & 0 & 0 & 0 & 0 & 10 & 72 & 138 & 62 & 89 & 0 & 0 & 0 & 0 & 0 & 0 & 0 & 0 & 0 \\
P5'_Emisison & 0 & 0 & 0 & 0 & 0 & 0 & 0 & 0 & 0 & 0 & 0 & 0 & 121 & 0 & 0 & 0 & 0 & 0 \\
P5_Emisison & 0 & 0 & 0 & 0 & 0 & 0 & 0 & 0 & 0 & 0 & 0 & 0 & 0 & 0 & 0 & 0 & 0 & 151 \\
P15"_Emisison & 0 & 0 & 0 & 0 & 0 & 0 & 0 & 0 & 0 & 0 & 0 & 0 & 0 & 0 & 0 & 0 & 80 & 0 \\
P5"_Emisison & 0 & 0 & 0 & 0 & 0 & 0 & 0 & 0 & 0 & 0 & 0 & 0 & 0 & 0 & 105 & 0 & 0 & 0 \\
P8_Emisison & 0 & 0 & 0 & 0 & 0 & 0 & 0 & 0 & 0 & 172 & 0 & 0 & 0 & 0 & 0 & 0 & 0 & 0 \\
P3_Emisison & 0 & 0 & 0 & 0 & 0 & 0 & 0 & 0 & 0 & 0 & 166 & 0 & 0 & 0 & 0 & 0 & 0 & 0 \\
P6_Emisison & 0 & 0 & 0 & 0 & 0 & 0 & 0 & 0 & 0 & 0 & 0 & 0 & 0 & 127 & 0 & 0 & 0 & 0 \\
P8"_Emisison & 0 & 0 & 0 & 0 & 0 & 0 & 0 & 0 & 0 & 0 & 0 & 0 & 0 & 0 & 0 & 100 & 0 & 0 \\
\hline
\end{tabular}

Figure 13. Emission cost incidence matrix.

\section{Evaluation}

The objective of our simulation-based evaluation is to analyze the performance of different vehicle routing methodologies. We will specifically analyze the question of: Which routing methodology offers the driver the most improved performance in terms of energy consumption, emissions, and travel time?

Two approaches were evaluated to support the premise of the research: the implementation of a physical experiment and computer-based simulation. Because the actual implementation of such a project is expensive due to the cost of equipping multiple vehicles and infrastructure with DSRC technology and because software programming is a trustworthy tool to perform accurate simulations, a computer-based simulation can avoid the time and expenses that accompany physical implementations. Thus, a computer-based proof-of-concept simulation is the tool of choice for our research.

For the vehicle routing application to be simulated, two simulators must be running simultaneously, namely, a traffic simulator and network simulator, with each simulator meeting a specific set of requirements and communicating in real time. The traffic simulator should be a microscopic traffic simulator, i. e., able to provide information about an individual vehicle, such as location, speed, acceleration, route, and travel time. Furthermore, the traffic simulator should be able to import real-world maps, such as TIGER or OSM, to provide the ability to simulate realistic traffic scenarios whose results can be reflected on the real world. Moreover, the traffic simulator should enable the incorporation of a vehicle fuel consumption and emission model that provides the opportunity to simulate the proposed eco-friendly routing application.

The network simulator should support wireless ad-hoc networks with dynamic topologies. Although different network simulators can provide those capabilities, it is also important that the network simulator support the new DSRC protocol stack used in vehicular networks for exchanging the various information with which cooperative decisions should be made. The coupling of the two simulators can be either open- or closed-loop coupling. The open-loop coupling [24] is a one-direction connection from the traffic simulator to network simulator. The traffic simulator is run first to generate mobility traces for the vehicles inside the simulation environment. These traces are then fed into the network simulator to simulate the exchange of messages between vehicle nodes. This interface is not suitable for our applications because these applications should be able to affect the behavior of the vehicles based on real-time events. The closed-loop coupling is a bidirectional connection between the two simulators running side by side. The positions of vehicles are continuously updated in the network simulator. A live application programming interface (API) socket connection allows for a real-time exchange of events between the two simulators. Such an interface satisfies the requirements needed to simulate ITS applications. However, only a few simulation platforms adopt this type of interface, such as the IntelliDrive Simulation Environment developed at the University of Virginia [25], VGSim [26], the Vehicles in Network Simulation (Veins) [27], and the Integrated Wireless and Traffic Simulation Platform for Real-time Road Traffic Management Solutions (iTETRIS) funded by the European Union [28]. Because ITS applications are only now being introduced in the industry and a long-term evolution simulation platform is preferred, the most-funded ( $€ 4.42$ million) simulation platform, iTETRIS, was pursued in the interest of continued tool maintenance and support. iTETRIS proposes an open-source platform, a flexible three-block simulation architecture, and real-time closed-loop coupling 
between the traffic simulator, Simulation of Urban Mobility (SUMO)[29], and the Network Simulator 3 (ns-3) [30].

A. Simulation Scenario

To validate the proof of concept of the proposed predictive routing methodology, a set of simulation scenarios were carefully designed and constructed to represent real-world traffic network scenarios. The following experimental configuration settings were chosen:

1. Network map: A real-world map of Eichstätt City in Bavaria, Germany (illustrated in Figure 14) was selected. The map was exported from OpenStreetMap and converted to a SUMO format using the application "Netconvert". The SUMO network file describing the traffic network includes roadway information, such as lanes, roadway slope, traffic lights, junctions, and speed limits.

2. Vehicle trips and traffic flow: A random trip and traffic flow file is generated by developing and running a python script entitled "randomTrips. py". A set of trips (including origin/destination, edges, and departure time) is generated with a uniform random distribution. Trips are dispatched throughout the simulation at equal intervals of one time step starting from zero to 100 (i. e., a distinct trip is generated at each simulation time step, and a new vehicle using this trip is emitted every $100 \mathrm{~s}$ ). However, the number of vehicles that share the same trip is constrained to six, resulting in a total of 100 trips $\times 6$ vehicles $=600$ vehicles running in $1 \mathrm{~h}$ of simulation time.

3. Initial Routes: The initial path that connects the origin to its corresponding destination was computed under real-time traffic conditions. The simulation network and its vehicle movements are initialized with travel cost (travel time or emissions) as the edge weight and utilizing the proposed PN-based ACC algorithm to find the optimal path. These static routes have been generated using the modified SUMO tool DUAROUTER and include the automatic iteration to compute the presented dynamic re-routing once the simulation is started.

4. Hybrid Communication Platform: To implement and evaluate the proposed dynamic routing methodology, the simulation experiments are run in a state-of-the-art hybrid wireless DSRC-based communication environment that supports V2V and V2I for exchanging traffic information. The exchanged messages in our simulation environment are divided into two main types based on the purpose of the message's payload instead of on the communication type (V2V or V2I):

5. Cooperative Awareness Messages (CAMs): messages exchanged between vehicles (V2V) and from vehicles to RSUs (V2I). The broadcast area for CAM messages is dynamic, related to the mobile vehicle communication area, and set to $100 \mathrm{~m}$. CAM packets comprise two data classes:

6. Vehicle travel and drive profile, including vehicle data, such as speed, position, and direction

7. Communication profile: transmission frequency

8. Geographical Broadcast Messages (GEOBROADCASTC): messages sent from RSUs to vehicles (I2V). These messages are used to broadcast the PTCI as computed by RSUs to all vehicles in the communication range. The broadcast area is static, related to the predetermined communication radius of the RSU (set at $400 \mathrm{~m}$ ).

9. RSU: Due to the slow computational processing from the increased number of RSUs and limited hardware system specifications, only eight RSUs have been implemented. Six of the RSUs were assigned to traffic light signals, and the other two RSUs were assigned to main junctions. To ensure that the majority of navigated vehicles receive messages from the RSUs, these units were distributed over the center area of the city, covering the highly congested roads. Multi-hop communication is activated for all traffic data broadcasted from RSUs to compensate for the limited number of RSUs.

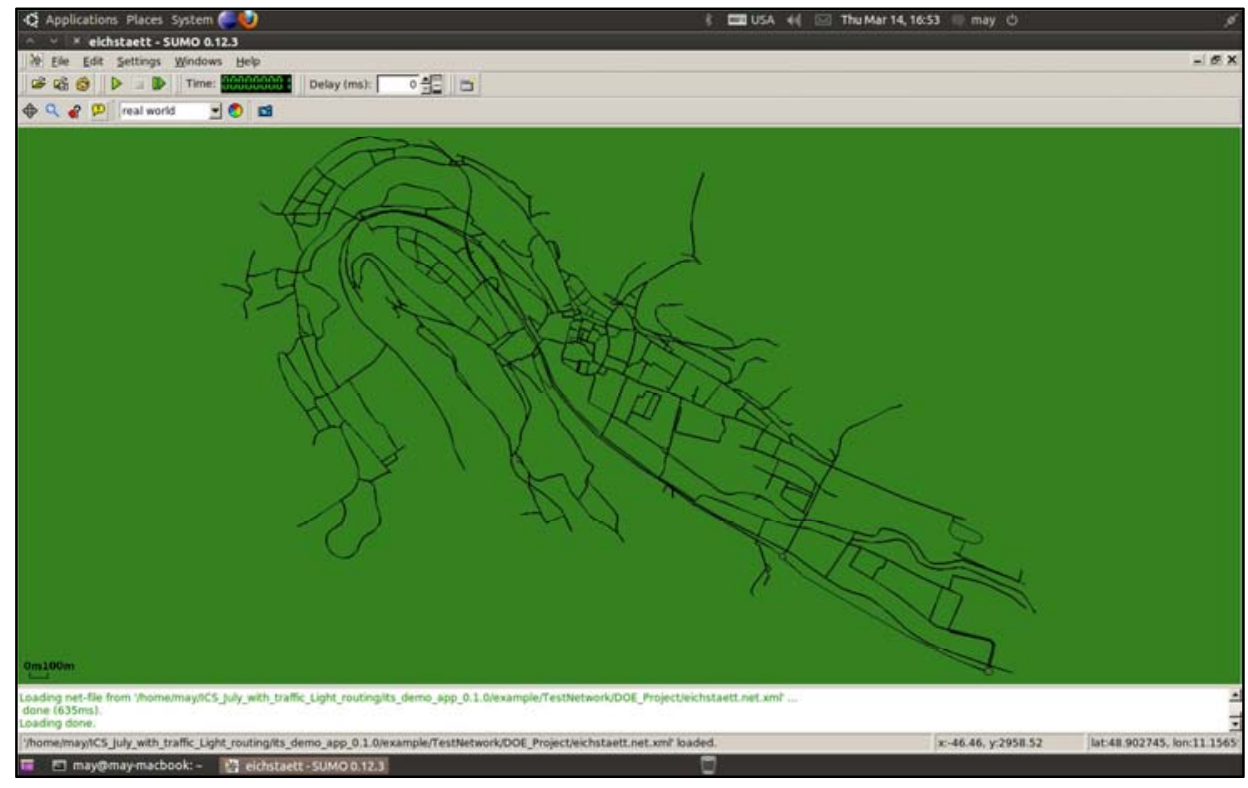

Figure 14. Map of Eichstätt City, Bavaria, Germany. 
B. Results and Analysis

Figure 15 and Figure 16 illustrate the advantages of an eco-static travel time-optimized routing in a congested traffic network with respect to the traditional shortest and fastest travel time-optimized routes.

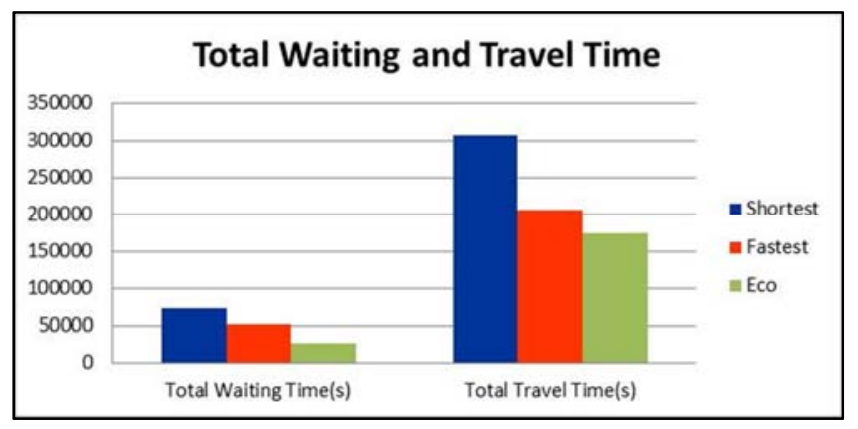

Figure 15. Shortest vs. fastest vs. eco (600 vehicles).

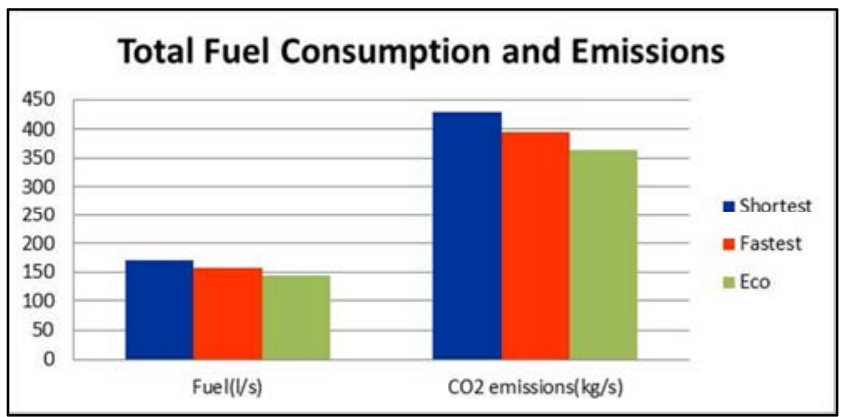

Figure 16. Shortest vs. fastest vs. eco (600 vehicles).

The results of the experiment are summarized in Table 4. The values are normalized to the result of the shortest-distance priority route. When comparing the three routing objectives, the eco-routing objective outperforms the shortest and fastest objectives in terms of all evaluated criteria.

Table 4. Comparison of experimental results for shortest vs. fastest vs. eco.

\begin{tabular}{lllll}
\hline Routing & Waiting Time & Travel Time & Fuel Consumption & CO $_{2}$ Emission \\
\hline Shortest & 1 & 1 & 1 & 1 \\
Fastest & 0.72 & 0.66 & 0.92 & 0.92 \\
Eco & 0.37 & 0.56 & 0.85 & 0.85 \\
\hline
\end{tabular}

Figure 17 and Figure 18 illustrate the further advantages of an eco-predictive travel time-optimized routing in a congested traffic network with respect to the traditional static and real-time based eco-optimized routing. In addition to the travel time, environmental performance improvements are achieved by reducing the fuel consumption and $\mathrm{CO}_{2}$ emissions. The travel time, fuel consumption, and $\mathrm{CO}_{2}$ emission improvements result from the predictive dynamic traffic status updates provided by the V2V and V2I communication infrastructure.

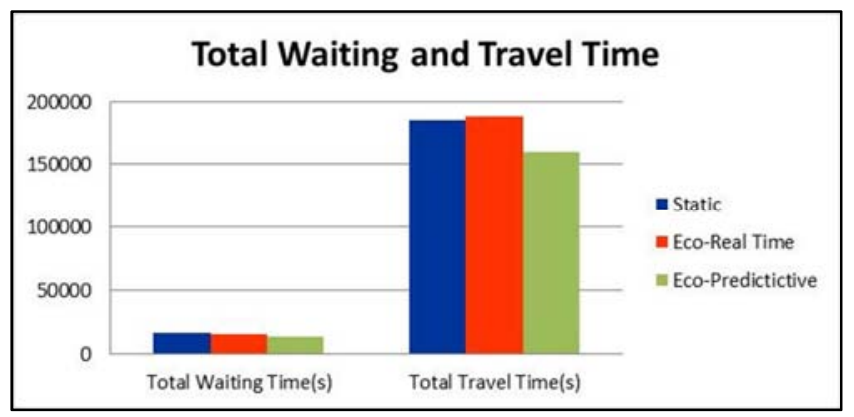

Figure 17. Static vs. eco real-time dynamic vs. eco predictive dynamic (600 vehicles).

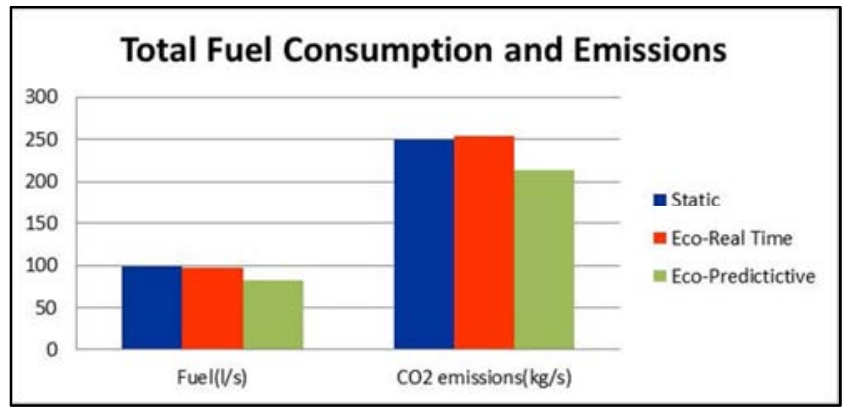

Figure 18. Static vs. eco real-time dynamic vs. eco predictive dynamic $(600$ vehicles).

The results of the experiment are summarized in Table 5 . The values are normalized to the results of the shortest-distance priority route. When comparing the results of the three routing objectives, the dynamic eco-predictive routing objective outperforms the dynamic eco-real time routing and static routing objectives. In this scenario, where traffic conditions are highly dynamic, real-time dynamic routing is rendered less effective compared to the static routing methodology due to the rapidly changing traffic conditions.

Table 5. Comparison of experimental results for shortest vs. fastest vs. eco.

\begin{tabular}{lllll}
\hline Routing & Waiting Time & Travel Time & Fuel Consumption & CO $_{2}$ Emissions \\
\hline Static & 1 & 1 & 1 & 1 \\
Real Time & 0.94 & 1.01 & 0.97 & 1.02 \\
Predictive & 0.81 & 0.86 & 0.83 & 0.85 \\
\hline
\end{tabular}

Experimentally, the resulting optimal route of the dynamic eco-predictive routing approach outperforms the static and time-dependent routing approaches in terms of accuracy and efficiency. We further evaluated the proposed predictive dynamic routing methodology simulating the highest level of foreseen traffic congestion, i. e., 1, 100 vehicles rather than 
600, while holding all other simulation parameters constant. Figure 19 and Figure 20 illustrate the same enhanced performance in travel time, fuel consumption, and $\mathrm{CO}_{2}$ emissions for the proposed dynamic predictive eco-routing methodology.

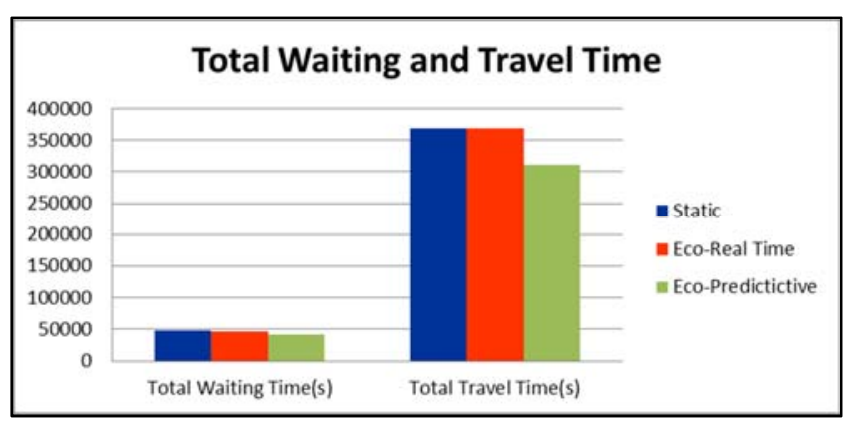

Figure 19. Static vs. eco real time dynamic vs. eco predictive dynamic $(1,100$ vehicles).

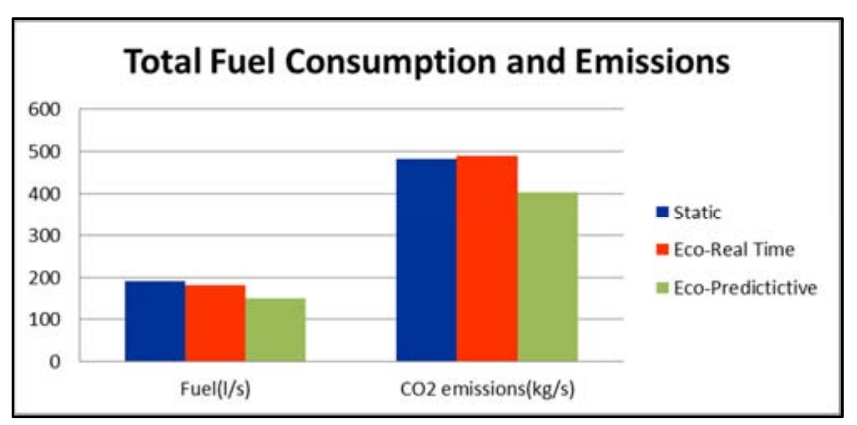

Figure 20. Static vs. eco real time dynamic vs. eco predictive dynamic $(1,100$ vehicles).

The results of the experiment are summarized in Table 6. The values are normalized to the results of the static distance priority route. When comparing the results of the three routing objectives, the eco-predictive routing outperforms the shortest and fastest objectives in all evaluated criteria.

Table 6. Comparison of experimental results for shortest vs. fastest vs. eco.

\begin{tabular}{lllll}
\hline Routing & Waiting Time & Travel Time & Fuel Consumption & CO $_{2}$ Emission \\
\hline Static & 1 & 1 & 1 & 1 \\
Real Time & 0.97 & 0.99 & 0.94 & 1.01 \\
Predictive & 0.88 & 0.84 & 0.78 & 0.83 \\
\hline
\end{tabular}

\section{Conclusion and Future Work}

ITS introduces a number of projects related to safety applications in vehicular environments. However, only a few applications have been proposed for reducing energy consumption and emissions. This paper proposes a vehicular routing methodology based on ITS infrastructure, illustrating the important role of vehicle routing methodologies in fuel consumption savings and emissions reduction. This paper exploits the relationships between route planning and travel time, energy consumption, and emissions reduction. This paper has demonstrated that a multivariable travel cost could be independently evaluated by developing and applying the unfolding PN methodology. The product of the work is an eco-predictive dynamic vehicle routing methodology that provides enhanced performance compared to all other routing methodologies. The second is an on-board and off-board data mining, computation and communication architecture providing a well-balanced approach addressing privacy, scalability, and, most importantly, computational time. The third product is an enhancement to the simulation tool iTETRIS that might be applicable for future related research. Presented evaluations of the environmentally friendly vehicle routing strategy based on predictive traffic information demonstrate a promising advantage over existing static and real-time based dynamic traffic information routing methodologies. According to our recent literature survey, no previous work has explored the potential of employing PN modeling in environmentally friendly vehicle routing utilizing a predictive traffic information tool. Our work lays the foundation for route planning in the unfolding space. The prediction of traffic conditions is an ambitious task. The performance of the traffic assessment model may be further enhanced through previous knowledge of when a traffic incident will be resolved. Another important aspect of the evaluation is to determine the precise relationship between the size of the unfolding PN and the planning problem, identifying properties that guarantee completeness of the finite route. These topics are relevant to further pursue performance enhancement for the predictive traffic assessment model. Extensive future research is required to develop and evaluate these benefits.

\section{References}

[1] National oceanic and atmospheric administration (NOAA), [Online],

"ftp://aftp.cmdl.noaa.gov/products/trends/co2/co2_annmean mlo.txt”, April 12, 2019, [April 15, 2019]

[2] Arrhenius, Svante, 1896. On the Influence of Carbonic Acid in the Air upon the Temperature of the Ground. Philosophical Magazine ser. 5, vol. 41, 237-276.

[3] U. S. Environmental Protection Agency, "Inventory of U. S. Greenhouse Gas Emissions and sinks: 1990-2017”, April 11 2019, EPA 430-R-19-001.

[4] U. S. Department of Transportation, [Online], "https://www.its.dot.gov", March 18, 2019, [April16, 2019]

[5] Fallouh. S; Abdul-Hak. M; Al-Holou. N, "Performance Evaluation of Data Communication Protocols in Intelligent Transportation", 2018 International Conference on Computer and Applications (ICCA), August 2018, DOI: 10.1109/COMAPP.2018.8460226

[6] U. S. Energy Information Administration, "Inventory of U. S Greenhouse Gas Emissions and Sinks: 1990-2016”, April 2018. 
[7] M. A. S. Kamal, Hayakawa, T., \& Imura, J.-i. (2018, Apr). Road-speed profile for enhanced perception of traffic conditions in a partially connected vehicle environment. IEEE Transactions on Vehicular Technology, vol. 67, no. 8, pp. 6824-6837, Aug. 2018.

[8] United States Department of Transportation - Federal Highway Administration, [Online],

"https://ops.fhwa.dot.gov/congestion_report_04/chapter2.htm", Feb 1, 2017, [April 17, 2019].

[9] P. Hao, G. Wu, K. Boriboonsomsin and M. J. Barth, "Eco-Approach and Departure (EAD) Application for Actuated Signals in Real-World Traffic," in IEEE Transactions on Intelligent Transportation Systems, vol. 20, no. 1, pp. 30-40, Jan. 2019. doi: 10.1109/TITS.2018.2794509

[10] S. Kidane Zegeye, B. De Schutter, H. Hellendoorn, E. Breunesse, "Reduction of Travel Times and Traffic Emissions Using Model Predictive Control" 2009 American Control Conference Hyatt Regency Riverfront, St. Louis, MO, USA June 10-12, 2009.

[11] Shahzada, "Dynamic vehicle navigation: An A* algorithm based approach using traffic and road information", Computer Applications and Industrial Electronics (ICCAIE), 2011 IEEE International Conference on Computer Applications and Industrial Electronics, Dec, 7, 2011, Page: 514-518

[12] Cascetta, E., A. Nuzzolo, F. Russo, and A. Vitetta (1996). "A Modified Logit Route Choice Model Overcoming Path Overlapping Problems: Specification and Some Calibration Results for Interurban Networks.” In J. B. Lesort (ed.), Transportation and Traffic Theory. Proceedings from the Thirteenth International Symposium on Transportation and Traffic Theory, Lyon, France, Pergamon pp. 697-711.

[13] C. Gawron. "An iterative algorithm to determine the dynamic user equilibrium in a traffic simulation model", International Journal of Modern Physics C, 9(3): 393-407, 1998

[14] Palubinskas, Gintautas and Kurz, Franz and Reinartz, Peter (2008) Detection of traffic congestion in optical remote sensing imagery. In: International Geoscience and Remote Sensing Symposium. IEEE. IGARSS08 , 2008-07-06 - 2008-07-11, Boston, USA.

[15] Cherrett, T., Waterson, B. and McDonald, M. (2005) Remote automatic incident detection using inductive loops. Proceedings of the Institution of Civil Engineers: Transport, 158, (3), PP. 149-155.

[16] J. H. Banks, "Introduction to transportation engineering. McGraw-Hill”, 2002.

[17] Bellman, Richard, "Adaptive Control Processes: A Guided Tour”, Princeton University Press, 1961

[18] S. Hausberger, M. Rexeis, M. Zallinger, R. Luz, "Emission Factors from the Model PHEM for the HBEFA Version 3", Report Nr. I-20/2009 Haus-Em 33/08/679 from 07.12.2009
[19] DLR,"An Integrated Wireless and Traffic Platform for Real-Time Road Traffic Management Solutions D3.1 - Traffic Modelling: Environmental Factors”, February, 13, 2009

[20] C. G. Cassandras and S. Lafortune, "Introduction to Discrete Event Systems" - Second Edition, Springer, 2008. ISBN 978-0-387-33332-8

[21] Ng, K. M.; Reaz, M. B. I.; Ali, M. A. M., "A Review on the Applications of Petri Nets in Modeling, Analysis, and Control of Urban Traffic," Intelligent Transportation Systems, IEEE Transactions on , vol. 14, no. 2, pp. 858, 870, June 2013

[22] McMillan, K. L.: Using unfoldings to avoid the state explosion problem in the verification of asynchronous circuits. In: Computer Aided Verification, 4th Inter- national Workshop (CAV'92). Volume 663 of Lecture Notes in Computer Science., Springer (1992) 164-177.

[23] Dijkstra, E. W. "A note on two problems in connection with graphs. Numerische Mathematik", 1959.

[24] D. B. Rehunathan, B. C. Seet, T. T. Luong, Federating of MITSIMLab and ns-2 for realistic vehicular network simulation, Proc. Mobility Conference 2007 - the 4th Int. Conf. Mobile Technology, Applications and Systems, Mobility 2007, Incorporating the 1st Int. Symp. Computer Human Interaction in Mobile Technology, IS-CHI 2007, p 62-67, 2007.

[25] H. Park, A. Miloslavov, J. Lee, M. Veeraraghavan, B. Park, B. L. Smith, Integrated Traffic/Communications Simulation Evaluation Environment for IntelliDriveSM Applications Using SAE J2735 Dedicated Short Range Communications Message Sets to be presented at the 2011 Annual Meeting of the Transportation, University of Virginia, United States, 2010.

[26] B. Liu, B. Khorashadi, H. Du , D. Ghosal,C. N. Chuah, M. Zhang, IVGSim: An integrated networking and microscopic vehicular mobility simulation platform" IEEE Communications Magazine, v 47, n 5, p 134-141, 2009.

[27] C. Sommer, Z. Yao, R. German, F. Dressler, On the need for bidirectional coupling of road traffic microsimulation and network simulation, Proc. 9th ACM International Symposium on Mobile Ad Hoc Networking and Computing (MobiHoc), p 41-48, 2008.

[28] Introduction to iTETRIS, [Online]," http://www.ict-itetris.eu/", [April 21, 2019]

[29] Daniel Krajzewicz, Jakob Erdmann, Michael Behrisch, and Laura Bieker. Recent Development and Applications of SUMO - Simulation of Urban Mobility. International Journal On Advances in Systems and Measurements, 5 (3\&4): 128-138, December 2012.

[30] Network Simulator, ns-3, [Online], "http://www.nsnam.org/", [April 21, 2019].

\section{Biography}

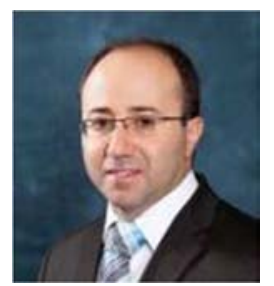

Mohamad Abdul-Hak (M’2008), received the B. S. in Electrical \& Computer Engineering from Wayne State University, Detroit, MI in 1999 and the M. S. degree in Electrical and Computer Engineering from University Of Michigan, Dearborn, MI in 2001. He received a Doctorate in Electrical \& Computer Engineering at the University of Detroit Mercy, Detroit, MI in 2013. Mohamad held multiple Electrical Engineering positons at General Motors and Chrysler Corporation before joining Mercedes Benz Research \& Development North America, Inc. in 2008 as a Senior Manager for the eDrive team. 


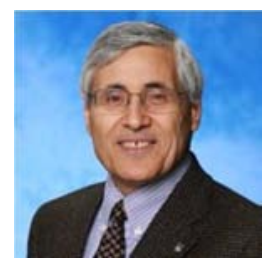

Nizar Al-Holou, is a Professor and a Chair of Electrical and Computer Engineering Department at the University of Detroit Mercy, Detroit, Michigan. His research interest is in the areas of Inter-vehicle and Intra-vehicle networking, Intelligent Transportation Systems (ITS), Distributed and parallel processing systems with an emphasis on automotive applications; Digital and Embedded systems. He received the B. S. in Electrical Engineering from Damascus University, Damascus, Syria in 1976, the M. S. degree in Electrical and Computer Engineering from Ohio State University Columbus, $\mathrm{OH}$ in 1983 and the Ph.D. in Electrical and Computer Engineering from University Of Dayton, Dayton, OH in 1987.

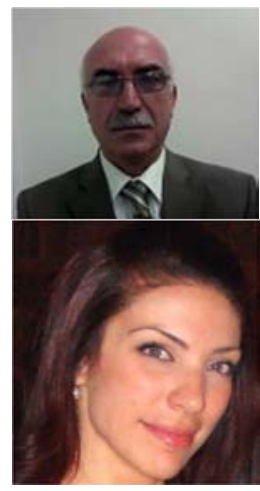

Youssef Ahmad Bazzi, received the B.S., M.S. and Ph.D. in Electrical \& Computer Engineering from Wayne State. He is currently a professor at the Lebanese University, Faculty of Engineering in Beirut, Lebanon. His research interests are in the areas of Sensor fusion, reasoning with uncertainty in the command control and counter measure systems, Predictive Routing, Vehicle Collision Avoidance Systems, Intelligent Transportation Systems.

Malok Alamir Tamer, received the B. S. in Information Technology from Aleppo University, Aleppo, Syria in 2005, the M. S. degree in Electrical and Computer Engineering from University of Detroit Mercy, Detroit, United States in 2013. 